\title{
Novel drug-delivery systems for patients with chronic rhinosinusitis
}

\author{
This article was published in the following Dove Press journal: \\ Drug Design, Development and Therapy \\ 30 May 2012 \\ Number of times this article has been viewed
}

\author{
Silviu Albu \\ Department of Otolaryngology, \\ University of Medicine and Pharmacy \\ Cluj-Napoca, Cluj-Napoca, Romania
}

Correspondence: Silviu Albu Department of Otolaryngology, University of Medicine and Pharmacy Cluj-Napoca, Str Republicii nr 18, 400015 Cluj-Napoca, Romania $\mathrm{Fax}+40264598278$

Email silviualbu63@gmail.com

\begin{abstract}
Chronic rhinosinusitis, one of the most common chronic medical complaints in the United States, seems to be increasing in incidence and prevalence, and has a significant impact on quality of life. Topical forms of medical therapy represent an attractive alternative for drug delivery to the nasal cavity and paranasal sinuses. Topical drug delivery has the advantage of directly acting on the site of inflammation, producing a higher concentration at the target site while avoiding systemic side effects. Although considerable research has been undertaken into improving nasal formulations in order to enhance absorption, little attention has so far been directed to upgrading the delivery devices. The aim of this review is to present current knowledge on the novel drug-delivery devices in use in the management of chronic rhinosinusitis patients, and to present the current available knowledge on topical drug penetration into the sinuses using various delivery devices. Additionally, methods used to enhance fluid sinus deposition are presented and the published clinical studies on the results of nebulized antibiotics in the treatment of chronic rhinosinusitis patients are discussed.
\end{abstract}

Keywords: paranasal sinuses, topical therapy, nebulized antibiotics, clinical trials

\section{Introduction}

Chronic rhinosinusitis (CRS) is one of the most common chronic medical complaints in the United States, affecting nearly $16 \%$ of the general population, and seems to be increasing in incidence and prevalence, accounting for 13 million physician visits annually and responsible for an estimated total cost of \$US6 billion/year. ${ }^{1,2}$

The etiology of CRS is thought to be multifactorial and can include bacterial infection, fungal colonization, environmental or aspirin sensitivities, immune or genetic disorders, poor mucociliary clearance, anatomic obstruction, and idiopathic inflammation. ${ }^{2,3}$ Chronic inflammatory disease of the paranasal sinuses was thought to arise from prolonged obstruction of the ostiomeatal complex, leading to stasis of secretions, mucociliary dysfunction, and failure to clear bacteria from the sinuses. ${ }^{2-4}$ Although there has been a shift to anti-inflammatory therapies in CRS, bacteria and fungi are still likely to be powerful mediators of inflammation. ${ }^{4}$ In the blocked sinus, bacteria that are normally removed from the sinuses by drainage of secretions may proliferate. The current model of CRS pathophysiology focuses on the interaction of the inflammatory mucosal disease with microbial flora and the failure of innate immunity. ${ }^{2-5}$

In the medical management of CRS, a combination of nasal saline irrigations, decongestants, nasal and systemic steroids, and courses of antibiotics are currently prescribed. $^{2-5}$ Current systemic agents, such as oral or intravenous antimicrobials 
or corticosteroids, have significant side effects and are not successful in many patients. This problem has led investigators to examine local delivery of topical therapies. ${ }^{6}$ In cases where conservative management has failed, endoscopic sinus surgery (ESS) is considered. ${ }^{3,4}$

Topical forms of medical therapy represent an attractive alternative for drug delivery to the nasal cavity and paranasal sinuses. Topical drug delivery has the advantage of directly acting on the site of inflammation, producing a higher concentration at the target site while avoiding systemic side effects. ${ }^{6}$ Moreover, a successful topical nasal treatment will increase the response rates of conservative management and will decrease the need for surgery. ${ }^{3,6}$ The general aim of topical therapy may also lie between potentially contending procedures such as mechanical irrigation and pharmaceutical intervention. ${ }^{5,6}$ The mechanical removal of pollutants, inflammatory products, mucus, antigen, and bacteria/biofilms is often addressed by nasal irrigation. These procedures rely on high-volume, positive-pressure solutions to provide shearing forces. However, the same approach may not be appropriate for drug delivery since complete sinus delivery, prolonged mucosal contact time with local absorption, and minimal depletion are often the desired properties. ${ }^{6}$

Corticosteroids and antibiotics have been successfully delivered through intranasal means. Various methods of topical drug delivery have been used, such as nasal drops, nasal sprays, nebulized droplets, and larger-volume irrigation., ${ }^{2,6}$ Despite general acceptance and clinical use of topical nasal therapy, only a few studies have concentrated upon the intranasal drug distribution. Although considerable research was carried out into improving nasal formulations in order to enhance absorption, little attention has so far been directed to upgrading delivery devices. The aim of the present review is to present the current knowledge on the novel drug delivery devices in use in the management of CRS patients, and to present the data on topical drug penetration into the sinuses. Additionally, methods used to enhance fluid sinus deposition are presented and the published clinical studies on the results of nebulized antibiotics in the treatment of CRS patients are discussed.

\section{Current nasosinusal topical drug delivery systems}

Nasal irrigation with isotonic or hypertonic saline is an inexpensive treatment and is a widely used drug delivery method in CRS patients. It is suggested that nasal irrigation removes inflammatory cells and excess secretions from the mucosal surface, decreases edema, enhances mucociliary clearance, and could be a potential route for topical drug delivery into the paranasal sinuses, including during the post-ESS period. ${ }^{6,7}$ However, it was demonstrated that access of the drug to the paranasal sinuses during nasal irrigation may only be possible post sinus surgery. ${ }^{8}$ Therefore, in addition to saline, other drugs, such as steroids and antibiotics, may be applied during nasal irrigation in the post-ESS period.

Another current topical treatment option is the use of nasal pump sprays, mainly because of their ease of use. These generate droplets between 50 and $100 \mu \mathrm{m}$ in diameter, and amounts between 70 and $150 \mu \mathrm{L}$ are administered per puff. Different drug formulations are available for use with nasal pump sprays, such as saline, decongestants, mucolytics, or steroids. However, a common feature of nasal sprays is the large fraction deposited in the anterior region of the nose with no significant aerosol access to the paranasal sinuses. ${ }^{9,10}$

The recently introduced breath-actuated bidirectional delivery device (OptiMist ${ }^{\mathrm{TM}}$; OptiNose AS, Oslo, Norway) surmounted several limitations of the current methods of nasal spray delivery. ${ }^{11}$ The OptiMist device has a conical, sealing nosepiece and a mouthpiece, includes a traditional spray pump and a breath-actuation mechanism, and generates droplets of $43 \mu \mathrm{m}$ diameter. The device is inserted into one nostril and the patient blows into the mouthpiece. Blowing closes the soft palate and transfers the oral pressure to the nostril. The nosepiece automatically balances the pressure in the nasal and oral cavities. In these instances, air is able to exit through the other nostril (bidirectional flow). Exhalation automatically triggers particle release at the moment where the positive dynamic pressure expands the nasal cavities. Since drug delivery occurs during exhalation, small particles cannot enter the lungs. ${ }^{11}$ Djupesland et a ${ }^{11}$ compared nasal deposition patterns between a conventional nasal spray and the OptiMist device. The deposition models generated by the two devices were compared in nine healthy subjects subsequent to technetium $99 \mathrm{~m}\left(\mathrm{Tc}^{99 \mathrm{~m}}\right)$ administration. ${ }^{11}$ The study demonstrated significantly larger initial and cumulative deposition in the upper posterior sector of the nasal cavities housing the middle meatus and sinus ostia and significantly lower deposition in the anterior segment.

Standard medical nebulizers can be used for aerosol generation and delivery into the nasal cavity. ${ }^{9}$ For efficient deposition into the sinuses, the aerosol should enter into the posterior nasal cavity. Two main nebulizer systems have been developed, differing in the diameter of the droplets produced and the flow patterns conveyed to these particles. ${ }^{12}$ Passive-diffusion nebulizers produce particles of a smaller size delivered in a constant direction; nevertheless, they have 
a slower velocity. Vortex-propelled nebulizers produce larger nebulized particles and centrifuge these droplets to the outer edge of the vortex represented by the walls of the nasal cavity and the paranasal sinuses. A passive-diffusion nebulizer system is the SinuNeb ${ }^{\mathrm{TM}}$ device (PARI Respiratory Equipment, Inc, Midlothian, VA). The SinuNeb device generates aerosolized particles of $3 \mu \mathrm{m}$ in diameter and delivers particles to the nasal cavity by passive diffusion through a hollow tube with two perforations at one end, spaced apart to match the nares. The nebulized liquid is then inspired transnasally. The ViaNase $^{\text {TM }}$ device (Kurve Technology Inc, Lynnwood, WA) is a vortex-propelled nebulizer system. The ViaNase device generates an active vortex of nebulized particles, contained within an occlusive nosepiece. The ViaNase device generates particles of between 9 and $11 \mu \mathrm{m}$ in size.

A recent development in the field of nebulizers is the pulsating aerosol delivery device..$^{9,10,13,14}$ A pulsating aerosol is an aerosol stream superimposed by a pulsation (sound wave). The German company PARI developed a commercial pulsating aerosol delivery device, the PARI Sinus ${ }^{\mathrm{TM}}$ Pulsating Aerosol System (PARI GmbH, Starnberg, Germany) in 2003. The PARI Sinus device contains the Pari LC Star jet nebulizer with a $3-\mu \mathrm{m}$ mass median aerodynamic diameter and output flow rate of $6 \mathrm{~L} /$ minute. A pulsation of $44 \mathrm{~Hz}$ is superimposed on the aerosol stream. Further developments incorporating a new vibrating membrane technology were recently reported, as commercialized in the eFlow ${ }^{\circledR}$ electronic nebulizer (PARI). Using this technology in the PARI device, the particle size distribution could be sharpened and the flow rate could be reduced to $3 \mathrm{~L} /$ minute. A pressure wave of $25 \mathrm{~Hz}$ frequency with amplitude of 20 mbar was superimposed on the aerosol stream. ${ }^{13,14}$

For delivery, the nebulizer is attached to one nostril and a flow resistor is plugged into the contralateral nostril. During delivery the patients are instructed to close their soft palate, which directs the aerosol from the delivery nostril to the second output nostril. Using this recommended protocol an aerosol pathway to the nasal airways is generated and drug penetration to the lung can be prevented. The output resistor and closure of the soft palate ensure optimal pressure transduction to the sinuses..$^{9,10,13,14}$

\section{Effectiveness of drug deposition into the sinuses}

The paranasal sinuses are air-filled cavities within the bones of the skull, ranging in volume between 5 and $30 \mathrm{~mL}$. The sinus is connected to the nasal cavity through the ostium, a narrow channel of about $1-3 \mathrm{~mm}$ in diameter and $10-15 \mathrm{~mm}$ in length. Since the sinuses are poorly ventilated, hollow cavities, current in vivo and in vitro experimentation has found inconsistent drug penetration into the sinuses.

The fundamental principles that determine the efficiency of deposition of aerosolized particles in the paranasal sinuses were described by Hyo et al. ${ }^{15}$ Using a plastic cast model, Hyo et al found that optimum particle size for deposition in the maxillary sinus varied with size of the ostia, and they documented no difference in deposition efficiency when comparing a plastic mold model and healthy volunteers. ${ }^{15}$ Mathematical modeling of aerosolized particle deposition suggests that three main factors are associated with particle penetration within the sinus: size of the ostium, pressure/ rate of flow of the aerosol, and particle size. Ostium size is the most significant factor. The authors concluded that ciliary action and respiration play a minor role in particle deposition, validating the use of a static cadaver model that has been used in subsequent studies of aerosolized delivery to the paranasal sinuses. ${ }^{15}$

Hyo et $\mathrm{al}^{15}$ theorized that the ideal particle size for aerosol delivery to the maxillary sinus would be $3-10 \mu \mathrm{m}$. However, an average of $3 \%$ of particles from 3 to $10 \mu \mathrm{m}$ in diameter penetrates into the maxillary sinus. On the other hand, Saijo et $\mathrm{al}^{16}$ investigated the particle deposition of steady-state aerosol flow in the paranasal sinuses in a post-ESS cast model. They found that an insertion angle of $45^{\circ}$ significantly increased the particle deposition compared with a $30^{\circ}$ insertion. They also showed that higher flow rates, smaller particle size, and larger ostial diameter allow for better penetration into the maxillary sinus. Particles of $5.63 \mu \mathrm{m}$ in diameter were deposited in the maxillary sinus in greater frequency than particles with a diameter of $16.37 \mu \mathrm{m} .{ }^{16}$

Negley et $\mathrm{al}^{17}$ studied the sinus deposition of technetium $\mathrm{Tc}^{99 \mathrm{~m}}$ administration by means of the RinoFlow ${ }^{\mathrm{TM}}$ nasal aerosol delivery device (Respironics, Inc, Cedar Grove, NJ) on five healthy subjects. The RinoFlow is a nebulizing device that delivers droplet particles in the $20-30 \mu \mathrm{m}$ range in a controlled flow. Subjects were asked to voluntarily perform the Politzer maneuver (occluding the alternate nostril and swallowing) during nebulization. Three of the five subjects revealed incomplete and inconsistent deposition in the frontal and maxillary sinuses. The sample size was too small to attain significance, but one should take into account that in subjects without any blockages of the ostiomeatal unit an insignificant amount of irrigation solution could be delivered into the paranasal sinuses. ${ }^{17}$

Olson et $\mathrm{al}^{18}$ also studied the distribution of nasal irrigation isotonic solutions in eight healthy, unoperated 
volunteers by comparing three-dimensional computed tomography imaging of three irrigation techniques: positivepressure irrigation, negative-pressure irrigation, and passivediffusion nebulization. Positive-pressure irrigation was generated by a squeeze bottle such as the Sinus Rinse ${ }^{\mathrm{TM}}$ (NeilMed, Inc, Santa Rosa, CA), negative pressure was created through sniffing and nebulization was generated by the RinoFlow device. The RinoFlow nebulizer was found ineffective, consistent with the previous study: ethmoid penetration was achieved in only two cases. Interestingly, nasal irrigation was effective in the cases of spray bottle use and inhalation methods. ${ }^{18}$ Contrast solution reached ethmoid and maxillary sinuses that were more uniform irrigated in positive-pressure administration. The ethmoid sinuses were penetrated in seven subjects during negative-pressure irrigation and in six subjects during positive-pressure irrigation. Maxillary sinus irrigation was highest in the positive-pressure group versus the negative-pressure group (seven versus five) with bilateral involvement in five of eight subjects. However, the study demonstrated universally poor sphenoid and frontal sinus penetration. According to these results, the ability of an irrigation technique to deliver a solution to the paranasal sinuses should be related to sufficient pressure and high volume. ${ }^{18}$

Wormald et $\mathrm{a} \mathrm{l}^{19}$ compared the effectiveness of three methods of nasal irrigation: irrigation with a nasal spray bottle, nasal nebulizer, and nasal douching bottle with the subject in the kneeling position. The study was performed on nine CRS patients after ESS and three normal subjects. Sinus deposition was assessed by radioactivity of $\mathrm{Tc}^{99 \mathrm{~m}}$ sulfur colloid. There was no statistical difference in measured radioactivity between the surgical and healthy subjects. Nasal douche was the most effective in both postsurgical and normal subjects. However, one limitation of nasal douching is that it may be difficult for older patients or patients with arthritis to adopt the position required. Nasal cavity, maxillary sinus, and the frontal recess (not frontal sinus) were the accessible areas, but no technique was found to reliably penetrate the sphenoid or frontal sinus. ${ }^{19}$

Snidvongs et $\mathrm{al}^{20}$ conducted a clinical study to investigate how well nasal irrigation could reach the paranasal sinuses in CRS patients. Fourteen patients, with bilateral CRS, underwent nasal irrigation with iodinated contrast solution. Nasal douching was carried out using an irrigation syringe in one side, and $10 \mathrm{~mL}$ of spray in the other. A computed tomography scan was undertaken for each patient to determine the volume and distribution of staining. Only two patients had any staining, with a small amount present in a total of three maxillary sinuses. The authors demonstrated that the two techniques had a similar performance. Both of them delivered only a small amount of the solution, if any, into the sinuses. Blockages of the ostiomeatal complex may be a primary cause for the poor sinus solution penetration. ${ }^{20}$

Hwang et $\mathrm{al}^{7}$ investigated ten healthy subjects and five post-ESS subjects looking at sinus penetration of the SinuNeb device, the ViaNase device, and the nasal spray bottle. Using radiolabeled saline, poor sinus penetration was seen with all three systems. The vortex nebulizer had the greatest potential for sinus penetration with rates of $30 \%$ penetration for the frontal sinus, $10 \%$ for the maxillary sinus, and $30 \%$ for the sphenoid sinus. In contrast, the SinuNeb device and the spray bottle both showed $0 \%$ penetration for all sinuses. The sinus penetration for the postoperative group was collectively poor. ${ }^{7}$

Valentine et $\mathrm{al}^{12}$ compared the sinus penetration of the PARI pulsed nebulizer to nasal irrigation with a squeeze bottle in a highly dissected cadaver model. Solutions were stained with methylene blue while staining intensity and colored area within the sinuses were assessed by three independent, blinded observers. The authors documented a significant increase in intensity of stain, percentage of stain, and circumference stained with the plastic nasal irrigation squeeze bottle versus the PARI device. Analysis of individual sinuses showed noteworthy increases in the indices of nasal douching relative to nebulization. In their study, $96 \%$ of all sinuses were stained by the squeeze bottle. The PARI device was noted to achieve ethmoid staining on a regular basis, whereas the other sinuses were inconsistently reached: frontal sinus, $43 \%$; maxillary sinus, $46 \%$, and sphenoid, $54 \% .^{12}$

In a recent study, Möller et a ${ }^{9,10}$ investigated sinus ventilation in healthy human volunteers using dynamic ${ }^{81 \mathrm{~m} K r-g a s ~ i m a g i n g ~ i n ~ c o m b i n a t i o n ~ w i t h ~ p u l s a t i n g ~ a i r f l o w s . ~}$ Ventilation is a basic requirement of aerosol delivery to the sinuses and subsequent deposition. ${ }^{81 \mathrm{~m}} \mathrm{Kr}$-gas was continuously ventilated through the nasal airways with and without pulsation and sinus gas access was monitored using gamma camera imaging. Without pulsation, only the central nasal cavity appeared on the image. With pulsation, the maxillary and frontal sinuses appeared on the gamma camera image. Without pulsation, less than $5 \%$ of the total $\mathrm{Kr}$-gas activity within the nasal cavity penetrated into the sinuses. This rate increased to about $48 \%$ with pulsation. In addition, pulsating airflow caused a sustained release of ${ }^{81 \mathrm{~m}} \mathrm{Kr}$-gas activity from the nasal cavity and the sinuses after switching off $\mathrm{Kr}$-gas delivery. The authors suggest that this postponement can 
cause an increased residence time of an aerosolized drug in the sinuses, further enhancing aerosol deposition..$^{9,10}$

During the same study, the deposition efficiency of $\mathrm{Tc}^{99 \mathrm{~m}}$-diethylene triamine pentaacetic acid aerosol delivered by pulsating airflow was assessed in each volunteer and compared to its deposition effectiveness using nasal pump sprays. A solution composed of $\mathrm{Tc}^{99 \mathrm{~m}}$-diethylene triamine pentaacetic acid was delivered to each nostril for 20 seconds. The authors compared their results on distribution and clearance with those obtained after nasal pump spray delivery of a similar $\mathrm{Tc}^{99 \mathrm{~m}}$-diethylene triamine pentaacetic acid solution. Nasal distribution was assessed by generating an image composed from lateral gamma camera images superimposed on a coronal magnetic resonance tomography scan of the volunteer. Nasal pump sprays gave anterior nasal airway deposition, and significant mucociliary clearance had already happened by the time the image was recorded. ${ }^{10}$ The pulsating aerosol delivery gave more posterior aerosol deposition, with access to the ostiomeatal complex. With pulsating aerosol delivery, the authors report that total deposition in the nasal cavity (including sinuses) of the five subjects was $71 \% \pm 17 \%$ of the nebulized dose, and $6.5 \% \pm 2.3 \%$ of the total nose activity (including sinuses) penetrated to the sinuses. In the same study it was demonstrated that by using the nasal pump spray, there was $100 \%$ deposition of the administered activity, and less than $1 \%$ of this dose penetrated into the sinuses. ${ }^{9}$

Aerosol delivered by nasal pump sprays has a high rate of clearance out of the nose: $50 \%$ was cleared after $14.2 \pm 3.4$ minutes, and after 6 hours, less than $5 \%$ of activity was retained in the nose. In contrast, the pulsating aerosol delivery has retarded clearance kinetics: $50 \%$ of the dose was cleared after $1.2 \pm 0.5$ hours, and more than $20 \%$ of the administered dose was retained in the nose after 6 hours..$^{9,10}$

The studies conducted by Möller et $\mathrm{al}^{9,10,13,14}$ have shown that drug delivery using pulsating aerosols was associated with slower clearance of the radiotracer from the nose compared with nasal pump spray delivery, suggesting penetration into the nose to sites with retarded mucociliary clearance. The authors conclude that clearance kinetics in the range of hours using pulsating aerosol delivery, compared with 10-20 minutes using nasal pump sprays, may provide longer residence times of a drug administered to the nose as a pulsating aerosol, and it may prevent rapid removal by mucociliary clearance and by circulation..$^{9,10,13,14}$ Therefore, pulsating aerosols may consent to formulations with sustained-release profiles and possibly once-daily application, even with shorter half-time values. ${ }^{9,14}$

\section{Methods used to enhance drug distribution within the sinuses}

Besides the delivery methods discussed previously, there are other methods used in clinical practice to enhance drug delivery to the sinuses: ESS and the position of the patient during nasal irrigation. ${ }^{6}$

Recent evidence supports the belief that ESS improves the delivery of topical medications to the sinus mucosa: Harvey et $\mathrm{al}^{8}$ studied the efficiency of nasal irrigation in ten cadavers and demonstrated that ESS significantly enhanced sinus delivery of medication, regardless of delivery device. ESS is essential to effectively allow topical distribution to the sinus mucosa. ${ }^{6,8,21}$ The frontal and sphenoid sinus are essentially inaccessible before surgery and according to Grobler et $\mathrm{al}^{22}$ an ostium size of greater than $4 \mathrm{~mm}$ is required to even begin drug penetration into the maxillary sinus. For those with mucosal edema and chronic inflammation, distribution is probably worse. For these reasons, Harvey and Schlosser $^{6}$ stated that in medically managing CRS, the use of expensive and time-wasting topical therapies, such as increasing topical steroid options, are probably not supported prior to ESS.

There are conflicting reports on the most effective position for drug delivery to the nasal cavity and paranasal sinuses. The majority of these studies involve assessment of dye distribution in the region of the middle turbinate with simple sprays and drops in nonoperated patients. ${ }^{23,24}$ Many commercial products recommend a head-down, over sink, with nose to ground position for irrigation. This orientation is practical and makes runoff easy to collect. One study demonstrated that the "Mygind" and "Ragan" (left lateral and supine positions) were superior to the "Mecca" and "Head Back" positions in delivery of drops to the middle meatus. ${ }^{25}$ However, these results were found inconclusive by other investigators. ${ }^{26-30}$ The relevance of positioning with positivepressure irrigation is supposed to be less significant. ${ }^{27-32}$ However, even with positive-pressure, high-volume irrigation, the head-down or lateral position may lead to better frontal distribution. ${ }^{33}$

\section{Clinical studies on the use of nebulized antibiotics in the management of CRS patients}

It is common practice to prescribe prolonged courses of antibiotics during the medical management of CRS patients. Although widely accepted, this strategy is empirical and based in part upon culture reports available. ${ }^{2-4}$ In many 
bacteriological culture studies, Staphylococcus aureus has been found to be a predominant species. ${ }^{3,4}$ Despite the consistency of culture results positive for $S$. aureus, there is no consensus on whether or not its presence is of pathogenic importance. The secretion of various enzymes and toxins feeds the inflammatory reaction and preserves the recalcitrance of the disease. Moreover S. aureus has been discussed in the literature because of its superantigen potentials and their apparent link to biofilm. ${ }^{3-5}$

Disadvantages of prolonged antibiotic administration include bacterial resistance, allergic reactions, nephrotoxicity, ototoxicity, and gastrointestinal and hepatic disturbances. It has been suggested that topical application of antibiotics directly to the target site prevents these adverse effects of prolonged systemic administration, and avoids selection of resistant gut microflora. On the other hand, topical antibiotics have the theoretical advantage of acting directly on the site of infection and producing a higher concentration of antibiotic at the target site. Such increased concentrations of topical antibiotics have also been shown to be effective in killing bacteria in biofilm form. ${ }^{3}$

There is experimental evidence on the effectiveness of topical therapy in the management of CRS. Recently, Antunes et $\mathrm{al}^{34}$ reported on the dose-dependent effects of topical tobramycin in an animal model of Pseudomonas aeruginosa sinusitis. They noted that as opposed to normal saline irrigations, topical tobramycin led to a significant improvement in the degree of infection in this animal model. ${ }^{34}$

It is interesting that so few clinical studies have been conducted to explore the therapeutic option of nebulized antimicrobials in the treatment of CRS. In a prospective study, Kamijyo et $\mathrm{al}^{35}$ reported on 28 patients treated with fosfomycin nebulization three times a week for a period of 4 weeks. Outcome measures included four subjective and five objective symptoms as well as cytokine concentrations. Improvement in terms of objective symptoms and endoscopic findings was rated as at least fair in about $60 \%$ of patients, except for the amount of secretion. Postnasal drip improved in $88 \%$ of the patients. ${ }^{35}$

Vaughan and Carvalho ${ }^{36}$ evaluated the effect of the nebulization of several antimicrobials over a period of 3 months in 42 patients with CRS. The authors compared the efficiency of six different culture-dependent nebulized antibiotics versus standard oral or intravenous antibiotic therapy. They reported significant improvements for posterior nasal discharge, facial pain, and emotional consequences. There was also an increase of the "disease-free interval period": an average of
17 weeks for nebulized therapy versus 6 weeks for standard therapy. ${ }^{36}$

In a retrospective evaluation, Scheinberg and Otsuji ${ }^{37}$ reported on the effect of nebulized antibiotics for the treatment of acute exacerbations of CRS in 41 patients. Eighty-three percent of the patients improved on nasal obstruction, facial pain, rhinorrhea, and malaise after administration of nebulized antibiotics. The researchers concluded that nebulized antibiotics should be considered for all patients with CRS who have undergone ESS and who have failed to respond to oral antibiotics or who do not tolerate them. ${ }^{37}$

Kobayashi and $\mathrm{Baba}^{38}$ used an ultrasound-type inhaler and studied therapy with aminoglycoside, fosfomycin, and cefmenoxime three times per week. Their findings suggested that in patients without previous sinus surgery, the main effect of the nebulized medications was in the nose, with only an indirect effect on the maxillary sinuses: no experimental evidence was found of antibiotic penetration into the maxillary sinus. ${ }^{38}$

In a randomized, double-blind trial Desrosiers and Salas-Prato ${ }^{39}$ reported that both tobramycin-saline solution and aerosolized saline solution led to equal improvements in quality of life, symptoms, and endoscopic aspects of the nasal mucosa. The addition of tobramycin appears of minimal benefit. ${ }^{39}$

Videler et $\mathrm{al}^{40}$ conducted a randomized, placebocontrolled, double-blind, cross-over pilot study on 14 patients with recalcitrant CRS. Nasal irrigation with bacitracin/ colimycin or placebo using the RhinoFlow nebulizer twice daily was administered in combination with oral levofloxacin. Outcome measures included symptomatic relief, quality of life scores, and endoscopic findings. The study demonstrated that in patients with therapy-resistant CRS, many symptoms have a tendency to decrease compared with the pretreatment period after using the nebulizer in combination with levofloxacin. None of the analyses comparing the bacitracin/ colimycin group with placebo, however, identified significant differences. Therefore this study has not confirmed any additional effect of the locally administered antibiotics. ${ }^{40}$

Emerging evidence from a recent review suggests that topical antibiotics may be useful as a treatment modality in CRS and its exacerbations. ${ }^{41}$ Current corroborating evidence, at a relatively low level of evidence, points to the efficacy of nasal irrigation or nebulization rather than delivery by nasal spray. For the antibacterial studies, the highest level of evidence currently exists for studies that have used postsurgical patients and culture-directed therapy. Both stable and 
acute exacerbations of CRS appear to benefit from topical antibiotics. $^{41}$

In the same review, it is suggested that topical antibiotics should not be first-line management but may be attempted in patients refractory to the traditional topical steroids and oral antibiotics. ${ }^{41}$

Larger and better-designed, randomized, double-blind, placebo-controlled trials are required to more fully evaluate this modality of treatment. In particular, the control group for these future studies should be an equivalent sinus placebo rather than alternative methods of therapy. Additional studies could also directly compare different delivery methods, surgical and nonsurgical patients, and culture-directed versus empiric treatment.

\section{Disclosure}

The author reports no conflicts of interest in this work.

\section{References}

1. Benninger MS, Ferguson BJ, Hadley JA, et al. Adult chronic rhinosinusitis: Definitions, diagnosis, epidemiology, and pathophysiology. Otolaryngol Head Neck Surg. 2003;129:S1-S32.

2. Rosenfeld RM, Andes D, Bhattacharyya N, et al. Clinical practice guideline: Adult sinusitis. Otolaryngol Head Neck Surg. 2007; 137 (Suppl 3):S1-S31.

3. Fokkens W, Lund V, Mullol J; for the European Position Paper on Rhinosinusitis and Nasal Polyps group. European position paper on rhinosinusitis and nasal polyps 2007. Rhinol Suppl. 2007;20:1-136.

4. Dykewicz MS, Hamilos DL. Rhinitis and sinusitis. J Allergy Clin Immunol. 2010;125:S103-S115.

5. Hatipoglu U, Rubinstein I. Anti-inflammatory treatment of chronic rhinosinusitis: a shifting paradigm. Curr Allergy Asthma Rep. 2008; 8:154-161.

6. Harvey RJ, Schlosser RJ. Local drug delivery. Otolaryngol Clin North Am. 2009;42(5):829-845.

7. Hwang PH, Woo RJ, Fong KJ. Intranasal deposition of nebulized saline: a radionuclide distribution study. Am J Rhinol. 2006;20:255-261.

8. Harvey RJ, Goddard JC, Wise SK, Schlosser RJ. Effects of endoscopic sinus surgery and delivery device on cadaver sinus irrigation. Otolaryngol Head Neck Surg. 2008;139:137-142.

9. Möller W, Lübbers C, Münzing W, Canis M. Pulsating airflow and drug delivery to paranasal sinuses. Curr Opin Otolaryngol Head Neck Surg. 2011;19(1):48-53.

10. Möller W, Schuschnig U, Khadem Saba G, et al. Pulsating aerosols for drug delivery to the sinuses in healthy volunteers. Otolaryngol Head Neck Surg. 2010;142:382-388.

11. Djupesland PG, Skretting A, Winderen M, Holand T. Breath actuated device improves delivery to target sites beyond the nasal valve. Laryngoscope. 2006;116:466-472.

12. Valentine R, Athanasiadis T, Thwin M, Singhal D, Weitzel EK, Wormald PJ. A prospective controlled trial of pulsed nasal nebulizer in maximally dissected cadavers. Am J Rhinol. 2008;22:390-394.

13. Möller W, Schuschnig U, Meyer G, Mentzel H, Keller M. Ventilation and drug delivery to the paranasal sinuses: studies in a nasal cast using pulsating airflow. Rhinology. 2008;46:213-220.

14. Möller W, Schuschnig U, Meyer G, et al. Ventilation and aerosolized drug delivery to the paranasal sinuses using pulsating airflow: a preliminary study. Rhinology. 2009;47:405-412.
15. Hyo N, Takano H, Hyo Y. Particle deposition efficiency of therapeutic aerosols in the human maxillary sinus. Rhinology. 1989;27:17-26.

16. Saijo R, Majima Y, Hyo N, Takano H. Particle deposition of therapeutic aerosols in the nose and paranasal sinuses after transnasal sinus surgery: a cast model study. Am J Rhinol. 2004;18:1-7.

17. Negley JE, Krause H, Pawar S, Reeves-Hoché MK. RinoFlow nasal wash and sinus system as a mechanism to deliver medications to the paranasal sinuses: results of a radiolabeled pilot study. Ear Nose Throat J. 1999;78(8):550-552, 553-554.

18. Olson D, Rasgon B, Hilsinger R. Radiographic comparison of three methods for nasal saline irrigation. Laryngoscope. 2002;112:1394-1398.

19. Wormald P-J, Cain T, Oates L, Wong I. A comparative study of three methods of nasal irrigation. Laryngoscope. 2004;114:2224-2227.

20. Snidvongs K, Chaowanapanja P, Aeumjaturapat S, Chusakul S, Praweswararat $\mathrm{P}$. Does nasal irrigation enter paranasal sinuses in chronic rhinosinusitis? Am J Rhinol. 2008;22:483-486.

21. Chiu AG, Kennedy DW. Disadvantages of minimal techniques for surgical management of chronic rhinosinusitis. Curr Opin Otolaryngol Head Neck Surg. 2004;12:38-42.

22. Grobler A, Weitzel EK, Buele A, et al. Pre- and postoperative sinus penetration of nasal irrigation. Laryngoscope. 2008;118:2078-2081.

23. Homer JJ, Raine CH. An endoscopic photographic comparison of nasal drug delivery by aqueous spray. Clin Otolaryngol Allied Sci. 1998;23:560-563.

24. Homer JJ, Maughan J, Burniston M. A quantitative analysis of the intranasal delivery of topical nasal drugs to the middle meatus: spray versus drop administration. J Laryngol Otol. 2002;116:10-13.

25. Miller TR, Muntz HR, Gilbert ME, Orlandi RR. Comparison of topical medication delivery systems after sinus surgery. Laryngoscope. 2004;114(2):201-204.

26. Merkus P, Ebbens FA, Muller B, Fokkens WJ. The 'best method' of topical nasal drug delivery: comparison of seven techniques. Rhinology. 2006; $44: 102-107$

27. Karagama YG, Lancaster JL, Karkanevatos A, O'Sullivan G. Delivery of nasal drops to the middle meatus: which is the best head position? Rhinology. 2001;39:226-229.

28. Kayarkar R, Clifton NJ, Woolford TJ. An evaluation of the best head position for instillation of steroid nose drops. Clin Otolaryngol Allied Sci. 2002;27:18-21.

29. Aggarwal R, Cardozo A, Homer JJ. The assessment of topical nasal drug distribution. Clin Otolaryngol Allied Sci. 2004;29:201-205.

30. Tsikoudas A, Homer JJ. The delivery of topical nasal sprays and drops to the middle meatus: a semiquantitative analysis. Clin Otolaryngol Allied Sci. 2001;26:294-297.

31. Cannady SB, Batra PS, Citardi MJ, Lanza DC. Comparison of delivery of topical medications to the paranasal sinuses via "vertex-to-floor" position and atomizer spray after FESS. Otolaryngol Head Neck Surg. 2005; 133:735-740.

32. Benninger MS, Hadley JA, Osguthorpe JD, et al. Techniques of intranasal steroid use. Otolaryngol Head Neck Surg. 2004;130:5-24.

33. Beule A, Athanasiadis T, Athanasiadis E, Field J, Wormald PJ. Efficacy of different techniques of sinonasal irrigation after modified Lothrop procedure. Am J Rhinol Allergy. 2009;23:85-90.

34. Antunes MB, Feldman MD, Cohen NA, Chiu AG. Dose-dependent effects of topical tobramycin in an animal model of Pseudomonas sinusitis. Am J Rhinol. 2007;21:423-427.

35. Kamijyo A, Matsuzaki Z, Kikushima K, et al. Fosfomycin nebulizer therapy to chronic sinusitis. Auris Nasus Larynx. 2001;28:227-232.

36. Vaughan WC, Carvalho G. Use of nebulized antibiotics for acute infections in chronic sinusitis. Otolaryngol Head Neck Surg. 2002;127:558-568.

37. Scheinberg PA, Otsuji A. Nebulized antibiotics for the treatment of acute exacerbations of chronic rhinosinusitis. Ear Nose Throat J. 2002; 81:648-652.

38. Kobayashi T, Baba S. Topical use of antibiotics for paranasal sinusitis. Rhinology Suppl. 1992;14:77-81. 
39. Desrosiers MY, Salas-Prato M. Treatment of chronic rhinosinusitis refractory to other treatments with topical antibiotic therapy delivered by means of a large-particle nebulizer: results of a controlled trial. Otolaryngol Head Neck Surg. 2001;125:265-269.

40. Videler WJ, van Drunen CM, Reitsma JB, Fokkens WJ. Nebulized bacitracin/colimycin: a treatment option in recalcitrant chronic rhinosinusitis with Staphylococcus aureus? A double-blind, randomized, placebo-controlled, cross-over pilot study. Rhinology. 2008;46(2):92-98.
41. Lim M, Citardi MJ, Leong JL. Topical antimicrobials in the management of chronic rhinosinusitis: a systematic review. Am J Rhinol. 2008; 22(4):381-389.

\section{Publish your work in this journal}

Drug Design, Development and Therapy is an international, peerreviewed open-access journal that spans the spectrum of drug design and development through to clinical applications. Clinical outcomes, patient safety, and programs for the development and effective, safe, and sustained use of medicines are a feature of the journal, which has also been accepted for indexing on PubMed Central. The manuscript management system is completely online and includes a very quick and fair peer-review system, which is all easy to use. Visit http://www.dovepress.com/testimonials.php to read real quotes from published authors.

Submit your manuscript here: http://www.dovepress.com/drug-design-development-and-therapy-journal 